\title{
Reviewer Acknowledgements for Global Journal of Health Science, Vol. 9, No. 3
}

Global Journal of Health Science wishes to acknowledge the following individuals for their assistance with peer review of manuscripts for this issue. Their help and contributions in maintaining the quality of the journal are greatly appreciated.

Global Journal of Health Science is recruiting reviewers for the journal. If you are interested in becoming a reviewer, we welcome you to join us. Please find the application form and details at http://recruitment.ccsenet.org and e-mail the completed application form to gjhs@ccsenet.org.

\section{Reviewers for Volume 9, Number 3}

\begin{tabular}{|c|c|c|}
\hline Abdulbari Bener & Ihn Sook Jeong & Pedram Iranmanesh \\
\hline Abin Varghese & Jan Chrastina & Pi-Ming Yeh \\
\hline Abiodun Adeniran & Jennifer Johnson & Pradnya KAKODKAR \\
\hline Aedan Olaso & Jo Ann Bamdas & Rami El Assal \\
\hline Ainize Peña Sarrionandia & Jose R. Cordon & Raphaële GIRARD \\
\hline Ali Javadi & Kinley Wangdi & Raymond Compton Jagessar \\
\hline Ama P. Fenny & Leandro Alberto Calazans Nogueira & Robert Sloan \\
\hline Amir Amani & Leena Kunnath Chacko & Rohit Rattan \\
\hline Angus N. Oli & Liu Liu & Roman Levytskyy \\
\hline Aris Gkoulalas-Divanis & Lizziane Winkelstroter & Sara Melo \\
\hline Asad Ali Khan Afridi & Loray Daws & Shaillay Dogra \\
\hline Camille Parsons & Lucy Fernandes & Somyeh Ahmad \\
\hline Carlos Martin Ardila M. & Marcel Wullschleger & Soon Soo Hoo \\
\hline Chidananda Mudalagiriyappa & Marian Kjellevold & Srikrishna Ramachandra \\
\hline Chung-Yu Chen & Marin Strøm & Suleyman Gorpelioglu \\
\hline Dongxu Fu & Maulik Parekh & Tomás Goicoa \\
\hline Evanthia Sakellari & Millie Parsons & Tsan Yang \\
\hline Faik Ardahan & Mini Sood & Usman Khan \\
\hline Farahnaz Amini & Mohammad Ahadi & Xiaosun Lu \\
\hline Gavric Zivana & Montarat Thavorncharoensap & Yan Ma \\
\hline Giovana Fernandes & Natasha AZZOPARDI MUSCAT & Yasam Kemal Akpak \\
\hline Hamid Hussain Madni & Nishith Vayada & Yongmei Huang \\
\hline Hülya YARDIMCI & Panayiotis C Petrou & \\
\hline
\end{tabular}

